Internist 2021 - 62:672-678

https://doi.org/10.1007/s00108-020-00930-w

Angenommen: 8. Dezember 2020

Online publiziert: 7. Januar 2021

(c) Springer Medizin Verlag GmbH, ein Teil von Springer Nature 2021

\section{Redaktion}

H. Haller, Hannover (Schriftleitung)

B. Salzberger, Regensburg

C.C. Sieber, Nürnberg

\section{Anamnese}

Die Aufnahme des 71 Jahre alten Patienten auf die Intensivstation erfolgte bei qualitativer Bewusstseinsstörung, Tachypnoe und Tachykardie mit arterieller Hypotonie unter dem Verdacht einer Sepsis. Ein zerebelläres Syndrom mit Intentionstremor und Ataxie, einer links betonten proximalen Parese sowie einer kognitiven Beeinträchtigung, das bei bekanntem fragiles-X-assoziiertem Tremor-Ataxie-Syndrom (FXTAS) vorbestand, hatte sich in den letzten Tagen laut Ehefrau verschlechtert. In den Tagen vor Aufnahme hatte der Patient auch zunehmend weniger gegessen und getrunken. Wegen autonomer Dysfunktion und benigner Prostatahyperplasie war der Patient mit einem suprapubischen Blasenkatheter versorgt.

Im Jahr 2010 war bei dem Patienten ein Diabetes mellitus Typ 2 diagnostiziert worden. Deswegen nahm der übergewichtige Patient (Body-Mass-Index [BMI] $36 \mathrm{~kg} / \mathrm{m}^{2}$ ) ein Kombinationspräparat mit Metformin und Sitagliptin ein und applizierte einen Glucagon-likepeptide-1(GLP-1)-Rezeptor-Agonisten. Etwa 6 Monate vor stationärer Aufnahme war die antidiabetische Therapie um Empagliflozin $25 \mathrm{mg} / \mathrm{Tag}$ ergänzt worden.

\author{
S. Großmann ${ }^{1}$ - U. Hoffmann ${ }^{2}$. C. Girlich ${ }^{2}$ \\ 'Klinik für Pneumologie und konservative Intensivmedizin, Krankenhaus Barmherzige Brüder \\ Regensburg, Regensburg, Deutschland \\ ${ }^{2}$ Klinik für Allgemeine Innere Medizin und Geriatrie, Krankenhaus Barmherzige Brüder Regensburg, \\ Regensburg, Deutschland
}

\title{
Bewusstseinsstörung, Tachypnoe und Tachykardie bei einem 71- jährigen Patienten mit Diabetes mellitus Typ 2
}

\section{Befund}

In der neurologischen Untersuchung imponierte ein somnolenter (Glasgow Coma Scale 11), dysarthrischer Patient mit Ruhetremor. Paresen fehlten. Die Atmung war beschleunigt (Atemfrequenz 24/min) und vertieft. Der Patient war tachykard (Herzfrequenz 105/min) und hypoton (systolischer Blutdruck $95 \mathrm{~mm} \mathrm{Hg}$ ), jedoch normotherm (Temperatur $36,8^{\circ} \mathrm{C}$ ). Klinisch fanden sich Zeichen der Exsikkose, allerdings kein Hinweis auf einen Infektfokus.

Die Urinuntersuchung ergab einen Nitrit-positiven Harnwegsinfekt, eine Glukosurie und eine Ketonurie (Ketonkörper semiquantitativ 2-fach positiv). Laborchemisch zeigten sich eine akute Nierenschädigung (geschätzte glomeruläre Filtrationsrate $46 \mathrm{ml} / \mathrm{min}$ ), eine Hyperglykämie (Blutzucker $242 \mathrm{mg} / \mathrm{dl}$, entspricht $13,4 \mathrm{mmol} / \mathrm{l}$ ) und nur minimal erhöhte Inflammationsparameter (Creaktives Protein [CRP] $19 \mathrm{mg} / \mathrm{l}$, Prokalzitonin $0,09 \mathrm{ng} / \mathrm{ml}$; Normbereiche: CRP $1-5 \mathrm{mg} / \mathrm{l}$, Prokalzitonin $0-0,05 \mathrm{ng} / \mathrm{ml}$ ).

In der Blutgasanalyse fiel eine ausgeprägte metabolische Azidose mit einem $\mathrm{pH}$ von 7,0 , einem Standardbikarbonat von $3,0 \mathrm{mmol} / 1$ und einer Anionenlücke $(\Delta \mathrm{AG})$ von $36 \mathrm{mmol} / \mathrm{l}$ auf.

Das Serumlaktat war nicht erhöht.
Diagnose

\section{- Euglykämische diabetische Keto- azidose (DKA) unter Therapie mit Natrium/Glukose-Kotransporter- 2(SGLT-2)-Inhibitor}

\section{Therapie und Verlauf}

Der Patient wurde auf die Intensivstation aufgenommen, die oralen Antidiabetika abgesetzt. Zur Unterdrückung der Ketogenese wurde eine kombinierte Therapie mit Insulin- und Glukosezufuhr durchgeführt. Dabei wurde eine kontinuierliche Zufuhr von bis $\mathrm{zu} 12 \mathrm{IE} / \mathrm{h}$ Insulin (über Perfusor) bei - aufgrund der nur mäßig erhöhten Blutglukosewerte - gleichzeitiger intravenöser Glukoseapplikation von teilweise $35 \mathrm{~g} / \mathrm{h}$ nötig, um sukzessive die Ketogenese zu unterdrücken, die metabolische Azidose auszugleichen und die $\Delta \mathrm{AG}$ zu normalisieren. Kalium wurde bei Insulinapplikation frühzeitig substituiert, eine Natriumbikarbonatgabe wurde nicht notwendig ( $\Delta \mathrm{AG}>$ Standardbikarbonatdifferenz).

Trotz frühzeitiger Phosphatsubstitution (bereits $8 \mathrm{~h}$ nach Beginn der Insulintherapie) entwickelte der Patient eine Hypophosphatämie bis $0,2 \mathrm{mmol} / \mathrm{l}$, die aber klinisch inapparent blieb. Nach $48 \mathrm{~h}$ konnte der Patient auf die Allgemeinstation verlegt werden.

In Anbetracht der Stoffwechselentgleisung wurden sicherheitshalber - trotz des höheren Alters des Patienten - die 


\begin{tabular}{l|l|l|}
\hline $\begin{array}{l}\text { Tab. } 1 \text { Ketonkörper- und Antikörperbe- } \\
\text { stimmung }\end{array}$ & Wert & $\begin{array}{l}\text { Norm- } \\
\text { bereich }\end{array}$ \\
\hline $\begin{array}{l}\text { B-Hydroxybutter- } \\
\text { säure }(m g / d l)\end{array}$ & 14,00 & Bis 3,50 \\
$\begin{array}{l}\text { Glutamat- } \\
\text { Decarboxy- } \\
\text { lase(GAD)-Anti- } \\
\text { körper im Serum } \\
\text { (IE/ml) }\end{array}$ & $<0,01$ & Bis 10,00 \\
\hline
\end{tabular}

Glutamat-Decarboxylase(GAD)-Antikörper zum Ausschluss eines latenten Autoimmundiabetes im Erwachsenenalter („latent autoimmune diabetes in adults" [LADA]) bestimmt (• Tab. 1).

Die Therapie des Typ-2-Diabetes wurde nach Erholung der Nierenfunktion dort und im weiteren Verlauf auch ambulant mit einem Kombinationspräparat aus Metformin und Gliptin sowie intensivierter Insulintherapie fortgeführt.

\section{Diskussion}

\section{Inhibitoren des Natrium/Glukose- Kotransporters 2}

SGLT-2-Inhibitoren wurden ab 2013 zur Therapie des Typ-2-Diabetes aufdem USamerikanischen Markt eingeführt [1]. In Deutschland stehen Dapagliflozin, Empagliflozin und Ertugliflozin zur Verfügung (Letzteres nur in Kombination mit Sitagliptin). Diese sogenannten Gliflozine finden auch in Deutschland eine zunehmende Verwendung in der Therapie des Typ-2-Diabetes. Eingesetzt werden sie in einer Kombinationstherapie mit weiteren Antidiabetika (beispielsweise Metformin) oder als Monotherapeutikum, falls Metformin nicht vertragen wird. Die von ihnen induzierte Hemmung der renalen Glukoserückresorption führt zu einer Glukosurie mit konsekutiver Reduktion der Blutzuckerspiegel.

Die hierdurch induzierte osmotische Diurese in Verbindung mit einer anhaltenden Natriurese aufgrund der SGLT2-abhängig reduzierten Natriumrückresorption im proximalen Nierentubulus ist wohl für die zusätzlich beobachteten kardio- und renoprotektiven Effekte mitverantwortlich. So fanden sich unter Therapie mit SGLT-2 Inhibitoren ein vermin- derter Progress der diabetischen Nephropathie und verminderte Harnsäurewerte [2].Die EMPA-REG-OUTCOME-Studie [3] konnte unter Empagliflozin eine Minderung des relativen Risikos für die kardiovaskuläre Mortalität um $38 \%$, für die Hospitalisation wegen dekompensierter Herzinsuffizienz um $35 \%$ und für den Tod jeglicher Ätiologie um $32 \%$ zeigen. Ähnliche Effekte wurden auch für andere SGLT-2-Inhibitoren gezeigt, unter anderem für Dapagliflozin in der DAPAHF-Studie [4]. Bezüglich der kardioprotektiven Effekte wird auch eine Superfuel-Theorie diskutiert. Hier wird postuliert, dass durch die Rückresorption von Ketonkörpern und deren Utilisation ein „Supersubstrat“ für das Myokard mit günstiger Energiebilanz entsteht [5]. In einer 2020 veröffentlichten Studie konnte der kardioprotektive und renoprotektive Effekt auch für die Anwendung bei Patienten ohne Diabetes mellitus gezeigt werden [6].

\section{》) Unter SGLT-2-Inhibitoren werden auch kardio- und renoprotektive Effekte beobachtet}

SGLT-2-Inhibitoren werden in der Leber glukuronidiert und unterliegen kombinierten Ausscheidungsmechanismen (renal/intestinal). Bei Niereninsuffizienz kann der Medikamentenspiegel als Ausdruck einer verminderten renalen Clearance über die Zeit um bis zu $48 \%$ erhöht sein. Bei einer glomerulären Filtrationsrate unter $45 \mathrm{ml} / \mathrm{min}$ ist aufgrund des Wirkmechanismus von einem abnehmenden therapeutischen Effekt auszugehen, außerdem besteht aufgrund des Risikos vermehrter Nebenwirkungen eine Kontraindikation für die Anwendung ([7]; • Abb. 1).

\section{Diabetische Ketoazidose}

Bei der DKA finden sich definitionsgemäß folgende pathologisch veränderte Laborparameter:

- Blutglukose $>250 \mathrm{mg} / \mathrm{dl}$

- Metabolische Azidose, $\mathrm{pH}<7,3$, $\mathrm{HCO}_{3}{ }^{-}<15 \mathrm{mEq} / \mathrm{ml}$
- Vergrößerte $\Delta \mathrm{AG}$

- Ketonkörper im Urin oder Serum

Die Schweregradeinteilung erfolgt über den $\mathrm{pH}$, die Menge des Bikarbonats und die neurologische Symptomatik (schwer: $\mathrm{pH}<7,0$, Serumbikarbonat $<10 \mathrm{mEq} / \mathrm{l}$, Stupor bzw. Koma).

Ursächlich findet sich beim Diabetes mellitus Typ 1 ein absoluter Insulinmangel mit vermehrter Glukoneogenese und Glykogenolyse bzw. verminderter peripherer Glukoseaufnahme. Durch Erhöhung der Carnitin-Palmitoyl-Transferase-Aktivität kommt es zu einer vermehrten Lipolyse und somit zur Verstoffwechselung von Fettsäuren in der Leber mit Ketonkörperbildung [8].

Im Jahr 2015 warnten die Food and Drug Administration (FDA) und European Medicines Agency (EMA) vor der Gefahr einer DKA [1] unter Therapie mit SGLT-2-Inhibitoren. Hierbei können DKA mit oder ohne Blutzuckererhöhungen auftreten. Bei der euglykämischen DKA finden sich Blutglukosewerte $<250 \mathrm{mg} / \mathrm{dl}$. Sie wurde erstmals 1973 bei Patienten mit Typ-1-Diabetes beschrieben [9].

Bei der SGLT-2-Inhibitor-induzierten DKA finden sich in $70 \%$ der Fälle Blutzuckerwerte $<250 \mathrm{mg} / \mathrm{dl}$, in $30 \%$ liegen sie $<200 \mathrm{mg} / \mathrm{dl}$ [2]. Aufgrund der nur gering bis mäßig erhöhten Blutzuckerwerte besteht die Gefahr einer verspäteten Diagnose der Stoffwechselentgleisung.

Folgende Mechanismen zur Entstehung der Ketonämie mit nur geringer Blutzuckererhöhung unter SGLT-2-Inhibitoren werden diskutiert [2]

- Hemmung des Glukosetransports im proximalen Tubulus mit Glukosurie, konsekutiv kommt es zu einer Minderung der Insulinsekretion und so zu niedrigeren portalen Insulinspiegeln

- Direkte Stimulation der pankreatischen a-Zellen mit Zunahme des Plasmaglukagonspiegels

- Verminderte renale Ausscheidung von $\beta$-Hydroxybutyrat bzw. Acetoacetat 


\section{Diagnostik der diabetischen Ketoazidose unter SGLT-2- Inhibitoren}

Klinisch finden sich die typischen Zeichen einer DKA mit metabolischer Azidose und kompensatorischer Hyperventilation (Kussmaulatmung; [10]), gastrointestinalen Symptomen (Erbrechen, Pseudoperitonitis diabetica), Zeichen der Exsikkose und Bewusstseinsstörung. Bezeichnenderweise können die Blutzuckerwerte wie oben beschrieben nur moderat erhöht sein.

Durch die Berechnung der $\Delta \mathrm{AG}$ lässt sich eine Additionsazidose nachweisen, die bei normalen Serumlaktatspiegeln und Ausschluss einer Intoxikation eine Ketonämie als Ursache wahrscheinlich macht. Eine Sicherung durch Ketonkörpernachweis im Urin (beispielsweise mittels Ketostix ${ }^{\circledR}$, Ascensia Diabetes Care Deutschland, Leverkusen) ist nicht immer zielführend, da durch die vermehrte renale Ketonkörperrückresorption der Ketonkörpernachweis im Urin gestört sein kann. Günstig wäre eine direkte Bestimmung der Ketonkörper (insbesondere von $\beta$-Hydroxybutyrat) im Blut. Hier finden sich im Durchschnitt Werte um $9,1 \pm 0,85 \mathrm{mmol} / 1$ [8].

\section{Azidoseformen bei der SGLT-2- Inhibitor-induzierten diabetischen Ketoazidose}

In den meisten Fällen ist die sofortige quantitative Bestimmung der Ketonkörper im Blut nur begrenzt möglich. Hier kann die Berechnung der $\Delta \mathrm{AG}$ den Verdacht auf das Vorliegen von Ketonkörpern erhärten.

$$
\text { Anionenlücke }=\mathrm{Na}^{+}-\left(\mathrm{Cl}^{-}+\mathrm{HCO}_{3}{ }^{-}\right)
$$

Der Normalwert liegt zwischen 8 und $16 \mathrm{mmol} / \mathrm{l}$. Bei ausgeprägter $\mathrm{Hy}$ palbuminämie ist eine Korrektur des Normalwerts nötig (pro Abfall um $1 \mathrm{~g} / \mathrm{dl}$ Albumin Anstieg der $\Delta \mathrm{AG}$ um $2,5 \mathrm{mmol} / 1$ [11]).

Neben der Erhöhung der Ketonkörper kann zusätzlich das Laktat im Serum vermehrt sein. Dies ist bedingt durch eine verminderte Glukoseutilisation bzw. eine Minderperfusion im Rahmen der begleitenden Hypovolämie. Bei Vorliegen einer Komedikation mit Metformin kann dieses ursächlich für eine Laktaterhöhung sein.

Bei der Therapie mit SGLT-2-Inhibitoren kommt es zusätzlich $\mathrm{zu}$ einer Hemmung des $\mathrm{Na}^{+} / \mathrm{H}^{+}$-Transporters (NHE3) und damit zu renalen Bikarbonatverlusten. Es können also eine Azidose durch vermehrte Bildung von Ketonkörpern (erhöhte $\Delta A G$ ) und eine zusätzliche azidotische Komponente durch Bikarbonatverlust $\triangle \mathrm{HCO}_{3}^{-}$(hyperchlorämische Azidose) auftreten.

Typischerweise ist dann der Quotient $\Delta \mathrm{AG} / \Delta \mathrm{HCO}_{3}{ }^{-}<1$ [12]. Im klinischen Kontext fällt auf, dass unter Therapie trotz Ausgleichs der $\triangle \mathrm{AG}$ eine metabolische Azidose persistiert (Zeitdifferenz 24-48 h; [13]).

\section{Hypophosphatämie}

In der DKA kommt es durch die $\mathrm{pH}$ Verschiebung zu einer Verschiebung von Kalium, Magnesium und Phosphat nach extrazellulär, das heißt, initial gemessene Phosphatspiegel im Serum können noch normal sein. Durch die vorbestehende Glukosurie mit konsekutiver Phosphaturie ist es aber tatsächlich zu einer Phosphatverarmung des Organismus gekommen. Inwieweit die Medikation mit SGLT-2-Inhibitoren selbst zu erniedrigten Phosphatreserven führt, ist nicht geklärt [14].

Das Phosphatdefizit bei der DKA beträgt im Durchschnitt $1 \mathrm{mmol} / \mathrm{kgKG}$ [8]. Die Insulintherapie zur Behandlung der DKA führt zu einer vermehrten Aufnahme von Phosphat in die Zelle, durch die notwendige Flüssigkeitszufuhr kommt es zusätzlich zu einer Dilutionshypophosphatämie.

Die intrazelluläre Verarmung an Adenosintriphosphat (ATP; [15]) und die verminderte Bildung von 2,3-Diphosphoglyzerat mit reduzierter Abgabe von Sauerstoff an die Gewebe führen zu unterschiedlich ausgeprägten klinischen Symptomen ( Infobox 1). Problematisch bei der DKA kann eine muskulär bedingte Insuffizienz der Atempumpe werden, da diese zum Ausgleich der metabolischen Azidose maximal stimuliert ist. Eine Phosphatsubstitution muss bei entsprechender Klinik oder bei gemessenen Serumphosphatwerten unter $0,32 \mathrm{mmol} / \mathrm{l}$ erfolgen. Da Phosphat
Internist 2021 · 62:672-678

https://doi.org/10.1007/s00108-020-00930-w

(c) Springer Medizin Verlag GmbH, ein Teil

von Springer Nature 2021

\section{S. Großmann · U. Hoffmann · C. Girlich}

Bewusstseinsstörung, Tachypnoe und Tachykardie bei einem 71-jährigen Patienten mit Diabetes mellitus Typ 2

\section{Zusammenfassung}

Bei Patienten mit Diabetes mellitus Typ 2 muss im Falle einer unklaren Bewusstseinsstörung und/oder Bauchschmerzen eine Natrium/GlukoseKotransporter-2(SGLT-2)-Inhibitorinduzierte diabetische Ketoazidose in die Differenzialdiagnose einbezogen werden. Dabei können die Blutzuckerspiegel trotz ausgeprägter Azidose nur moderat erhöht sein. Bei Nachweis ist eine umgehende intensivmedizinische Therapie unerlässlich.

\section{Schlüsselwörter}

Diabetes mellitus Typ 2 - SäureBasen-Gleichgewicht · Ketoazidose . Natrium/Glukose-Kotransporter-2Inhibitoren · Hypophosphatämie

Confusion, tachypnea, and tachycardia in a 71-year-old man with type 2 diabetes mellitus

\section{Abstract}

Patients with type 2 diabetes who present with confusion and/or abdominal pains should be screened for sodium-glucose cotransporter 2 (SGLT-2)-induced diabetic ketoacidosis. Severe acidosis was diagnosed despite only moderately increased blood sugar levels. If so, immediate ICU treatment is essential.

Keywords

Diabetes mellitus type 2 - Acid-base equilibrium · Ketosis - Sodium-glucose transporter 2 inhibitors · Hypophosphatemia

hauptsächlich intrazellulär vorkommt, ist eine Dosisabschätzung schwierig. Anhaltspunkte bilden Empfehlungen mit initialer Dosis von 0,08 bis $0,16 \mathrm{mmol} /$ $\mathrm{kgKG}$ über $6 \mathrm{~h}$ bzw. 32 bis $64 \mathrm{mmol} / \mathrm{Tag}$ über 7-10 Tage zum Auffüllen der Speicher [15]. 


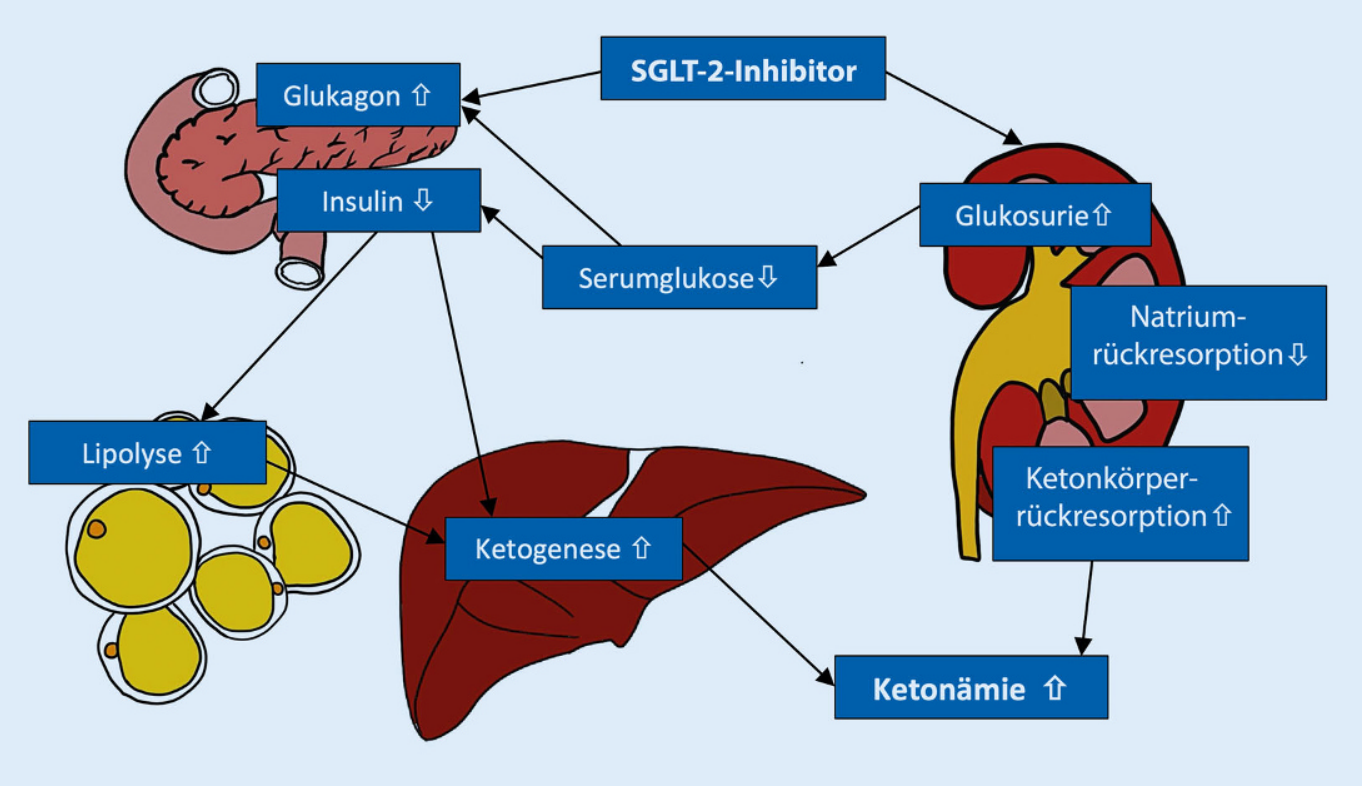

Abb. $1<$ Wirkung der SGLT-2-Inhibitoren auf Pankreas, Niere, Fettgewebe und Leber. SGLT-2 Natrium/Glukose-Kotransporter 2. (Modifiziert nach [2], Organe erstellt mit Procreate ${ }^{\circledR}$ )

\section{Risikofaktoren für die Ausbildung einer Ketoazidose unter SGLT-2- Inhibitoren $[1,2,17]$}

Eine Ketoazidose unter Einnahme von SGLT-2-Inhibitoren entsteht vermehrt dann, wenn die Kohlenhydratzufuhr vermindert ist, die Insulinsekretion gestört ist oder vermehrt kontrainsulinäre Hormone gebildet werden. Es besteht keine typische zeitliche Zuordnung zwischen Ersteinnahme des SGLT-2-Inhibitors und Auftreten einer DKA [18]. Über $20 \%$ der Patienten zeigen keinen detektierbaren Trigger.

Ein weiterer Risikofaktor ist die Anwendung von SGLT-2-Inhibitoren zur Insulineinsparung bei Patienten mit autoimmun bedingtem Diabetes mellitus. Hierzu gehören neben den Patienten mit Typ-1-Diabetes auch Patienten mit LADA. Deswegen sollten bei Patienten mit Diabetes und typischer Konstellation (Alter $<50$ Jahre, BMI $<25 \mathrm{~kg} / \mathrm{m}^{2}$, Autoimmunerkrankungen in der [Familien-] Anamnese) entsprechende Antikörperbestimmungen erfolgen, insbesondere eine Bestimmung der GAD-Autoantikörper [19].

\section{Prophylaxe einer diabetischen Ketoazidose unter Therapie mit SGLT-2-Inhibitoren}

Um das Auftreten einer DKA zu vermeiden, müssen die Risikofaktoren bzw. Auslöser (•Infobox 2) vermieden wer- den. In jedem Fall muss die Therapie mit SGLT-2-Inhibitoren bei Trauma, schweren Infektionen und $24 \mathrm{~h}$ vor elektiven Operationen beendet werden. Nach dem Auftreten einer SGLT-2-Inhibitor-assoziierten DKA ist eine Wiederaufnahme der Medikation wegen der Rezidivhäufigkeit kritisch zu sehen [20].

\section{Häufigkeit und Verlauf einer diabetischen Ketoazidose unter SGLT-2-Inhibitoren}

Eine DKA findet sich unter Therapie mit SGLT-2-Inhibitoren mit einer Häufigkeit von 0,16 bis $0,76 / 1000$ Patienten und Jahr [21]. Dabei ist das Auftreten bei Patienten mit Typ-2-Diabetes um den Faktor 2,2(-4) im Vergleich zur Anwendung von Dipeptidylpeptidase-4-Hemmern erhöht [22, 23]. Außerhalb kontrollierter Studien fand sich ein bis $\mathrm{zu}$ 7-fach erhöhtes Risiko [18]. Eine DosisNebenwirkungs-Beziehung ist bisher für keine der einzelnen Substanzen nachgewiesen. Es wurde gezeigt, dass ein „off label use" von Canagliflozin bei Patienten mit Typ-1-Diabetes das Risiko ketonkörperassoziierter Nebenwirkungen um 9,4\% erhöht [18].

Die Letalität der SGLT-2-induzierten DKA beträgt etwa $1,54 \%$ und ist somit im Vergleich zu DKA anderer Ursachen $(0,4 \%)$ erhöht [24].
Therapie $[2,8]$

Therapieempfehlungen bei DKA [25].

Bei der DKA im Rahmen eines Typ-1Diabetes wird eine Bolusapplikation von kurz wirksamem Insulin i.v. mit 0,1 IE/ kgKG gefolgt von einer kontinuierlichen Therapie mit $0,05-0,10 \mathrm{IE} / \mathrm{kgKG}$ pro $\mathrm{h}$ über Perfusor empfohlen. Ziel ist dabei, die Ketogenese $\mathrm{zu}$ hemmen und die $\triangle \mathrm{AG}$ sowie die metabolische Azidose zu normalisieren. Dabei soll der Blutzucker um maximal $50 \mathrm{mg} / \mathrm{dl}$ pro $\mathrm{h}$ abfallen, $\mathrm{ab}$ Blutzuckerwerten $<300 \mathrm{mg} / \mathrm{dl}$ wird daher die zusätzliche Infusion von Glukose 10-20\% i.v. empfohlen.

\section{》) Bikarbonat wird nur bei ausgeprägter Azidose mit $\mathrm{pH}<6,9$ gegeben}

Zum Ausgleich der Hypovolämie wird eine initiale Flüssigkeitszufuhr mit $\mathrm{NaCl}$ $0,9 \% 15-20 \mathrm{ml} / \mathrm{kgKG}$ pro h i.v. durchgeführt, die weiteren Infusionslösungen werden abhängig von den gemessenen Elektrolyten ausgewählt.

Wegen der Gefahr der Hypokaliämie wird frühzeitig (bei Serumkalium $<5,3 \mathrm{mmol} / \mathrm{l}$ ) mit einer Substitution von Kaliumchlorid begonnen. Bei einem Serumkalium $<3,3 \mathrm{mmol} / \mathrm{l}$ wird die Insulinzufuhr vorerst pausiert, bis die Hypokaliämie durch Substitution ausgeglichen ist. 
Infobox 1 Klinische Symptome bei Hypophosphatämie. (Nach [15, 16])

\section{- Zerebrale Anfälle}

- Tremor

- Parästhesien

- Guillain-Barré-ähnliche Neuropathien

- Quantitative Bewusstseinsstörungen

- Myokardiale Pumpfunktionseinschränkung

- Respiratorisches Versagen

- Dysphagie

- lleus

- Rhabdomyolyse (insbesondere bei Patienten mit chronischem Alkoholabusus)

- Muskuläre Schwäche

- Hämolyse

- Störung der granulozytären Chemotaxis (höhere Rate an Sepsis mit gramnegativen Erregern)

Da bei der klassischen DKA eine Additionsazidose (Ketonkörper) vorliegt, wird Bikarbonat nur bei ausgeprägter Azidose mit $\mathrm{pH}<6,9$ gegeben. Die empfohlene Dosis liegt bei 50 mmol Natriumbikarbonat über $1 \mathrm{~h}$. Bei Überdosierung besteht die Gefahr der Entwicklung einer Hypokaliämie, eines Hirnödems, einer paradoxen zerebralen Azidose und einer verminderten Abgabe von Sauerstoff an die Gewebe.

\section{》) Unter SGLT-2-Inhibitoren tritt eine diabetische Ketoazidose mit oder ohne Blutzuckererhöhungen auf}

Eine Phosphatsubstitution ist nur indiziert, wenn der Patient eine myokardiale Dysfunktion oder eine Erschöpfung der Atempumpe zeigt. Bei Serumwerten unter $0,32 \mathrm{mmol} / \mathrm{l} \mathrm{kann}$ unabhängig von klinischen Symptomen eine Substitution erwogen werden (s. oben).

Differenzialtherapie bei (euglykämischer) DKA unter Therapie mit SGLT-2Inhibitoren. Bei der DKA unter SGTL2-Inhibitoren sind die gemessenen Blutzuckerwerte relativ niedrig. Dennoch ist es aufgrund der Entstehungsmechanismen der Ketoazidose notwendig, die Lipolyse durch Insulingabe zu hemmen. Dies kann nur bei gleichzeitiger Kohlen-
Infobox 2 Triggerfaktoren für das Auftreten einer diabetischen Ketoazidose unter Natrium/ Glukose-Kotransporter-2Inhibitoren

- Alkoholkonsum/-abusus

- Zustand nach Pankreatitis/chronische Pankreatitis

- Chronische Lebererkrankung

- Erbrechen

- Dehydratation

- Exzessives Training

- Viraler oder bakterieller Infekt

- Operation (insbesondere bariatrische Operation mit postoperativ verminderter Kohlenhydrataufnahme)

- Trauma

- Thyreotoxikose

- Signifikante Reduktion einer Insulintherapie

hydratzufuhr mittels Glukoseinfusion erfolgen. Aufgrund der Osmolarität der Substanz ist es notwendig, dies über einen zentralen Venenkatheter zu tun.

Zielparameter ist ein kontinuierlicher Rückgang der $\triangle \mathrm{AG}$ in den initial stündlich durchzuführenden Blutgasanalysen, ohne dass es zu einem kritischen Abfall der Blutzuckerwerte kommt (s. oben).

Wegen der möglicherweise vorliegenden zusätzlichen hyperchlorämischen Azidose ist die Flüssigkeitszufuhr mit balancierten Elektrolytlösungen günstiger als die Infusion von $\mathrm{NaCl} 0,9 \%$ mit unphysiologisch hohem Chloridanteil [26]. Bei ausgeprägter Azidose (insbesondere bei $\Delta \mathrm{AG} / \Delta \mathrm{HCO}_{3}{ }^{-}<1$ ) ist auch die moderate Zufuhr von Bikarbonat gerechtfertigt.

Die metabolische Rekompensation kann zu einer ausgeprägten Hypophosphatämie führen. Regelmäßige Kontrollen sind dringend anzuraten, eine Substitution sollte bei klinischen Symptomen oder Serumphosphatwerten unter $0,32 \mathrm{mmol} / \mathrm{l}=1 \mathrm{mg} / \mathrm{dl}$ erfolgen ([17]; s. oben).

Das Behandlungsziel ist erreicht, wenn eine gute Stoffwechseleinstellung gegeben, die $\Delta \mathrm{AG}$ unter $12 \mathrm{mmol} / \mathrm{l}$ gesunken bzw. die metabolische Azidose ausgeglichen ist. Dieses Ziel wird bei dieser Form der DKA erst nach längerer intensivmedizinischer Behandlung erreicht ( 4 vs. 2 Tage; $p=0,019$; [27]); als Grund hierfür wird die verzögerte
Diagnosestellung, aber auch die schwer auszugleichende Azidose diskutiert.

\section{Fazit für die Praxis}

- Patienten unter Therapie mit einem Natrium/Glukose-Kotransporter2(SGLT-2)-Inhibitor, die eine schwere metabolische Azidose aufweisen, sollten trotz relativ niedriger Blutzuckerwerte auf eine Ketoazidose hin untersucht werden.

- Da die direkte Bestimmung von Ketonkörpern im Blut meist nicht realisiert werden kann, hilft die Bestimmung der Anionenlücke.

- Patienten mit SGLT-2-Inhibitorassoziierter diabetischer Ketoazidose benötigen eine intravenöse Insulintherapie mit gleichzeitiger Zufuhr von Glukose zur Hemmung der Ketoneogenese. Regelmäßige Phosphatbestimmungen sind dabei essenziell.

- In bestimmten Risikokonstellationen sollte der SGLT-2-Inhibitor pausiert werden. Dies ist aufgrund eigener klinischer Erfahrungen auch bei Patienten mit schwereren Coronavirusdisease-2019(COVID-19)-Verläufen und damit verbundener Anorexie und gleichzeitig vermehrter Atemarbeit bei pulmonaler Beteiligung zu beachten.

\section{Korrespondenzadresse}

\section{Dr. S. Großmann}

Klinik für Pneumologie und konservative Intensivmedizin, Krankenhaus Barmherzige Brüder Regensburg

Prüfeninger Straße 86, 93049 Regensburg, Deutschland

stefan.grossmann@barmherzige-regensburg.de

\section{Einhaltung ethischer Richtlinien}

Interessenkonflikt. S. Großmann, U. Hoffmann und C. Girlich geben an, dass kein Interessenkonflikt besteht.

Für diesen Beitrag wurden von den Autoren keine Studien an Menschen oder Tieren durchgeführt. Für die aufgeführten Studien gelten die jeweils dort angegebenen ethischen Richtlinien. Für Bildmaterial oder anderweitige Angaben innerhalb des Manuskripts, 
über die Patienten zu identifizieren sind, liegt von ihnen und/oder ihren gesetzlichen Vertretern eine schriftliche Einwilligung vor.

\section{Literatur}

1. Bonora BM, Avogaro A, Fadini GP (2018) Sodiumglucose co-transporter-2 inhibitors and diabetic ketoacidosis: an updated review of the literature. Diabetes Obes Metab 20(1):25-33

2. Diaz-Ramos A, Eilbert W, Marquez D (2019) Euglycemic diabetic ketoacidosis associated with sodium-glucose cotransporter-2 inhibitor use: a case report and review of the literature. Int J Emerg Med 12(1):27

3. Zinman Betal (2015) Empagliflozin, cardiovascular outcomes, and mortality in type 2 diabetes. N Engl JMed 373(22):2117-2128

4. McMurray JJV et al (2019) Dapagliflozin in patients with heart failure and reduced ejection fraction. NEngl J Med 381(21):1995-2008

5. Ferrannini E, Mark M, Mayoux E (2016) CV protection in the EMPA-REG OUTCOME trial: a "thrifty substrate" hypothesis. Diabetes Care 39(7):1108-1114

6. Packer M et al (2020) Cardiovascular and renal outcomes with empagliflozin in heart failure. NEngl J Med 383(15):1413-1424

7. Ingelheim B (2019) Fachinformation Jardiance (Rote Lise)

8. Kitabchi AE et al (2009) Hyperglycemic crises in adult patients with diabetes. Diabetes Care 32(7):1335-1343

9. Munro JF et al (1973) Euglycaemic diabetic ketoacidosis. Br Med J 2(5866):578-580

10. Gallo de Moraes A, Surani S (2019) Effects of diabetic ketoacidosis in the respiratory system. World JDiabetes 10(1):16-22

11. Kimmel M, Alscher MD (2016) Disorders of the acid-base balance and the anion gap. Dtsch Med Wochenschr 141(21):1549-1554

12. Rastegar A (2007) Use of the DeltaAG/DeltaHCO3ratio in the diagnosis of mixed acid-base disorders. JAm Soc Nephrol 18(9):2429-2431

13. Dizon $S$ et al (2017) Insights into the recognition and management of SGLT2-inhibitor-associated ketoacidosis: it's not just euglycemic diabetic ketoacidosis. Can J Diabetes 41 (5):499-503

14. Shoukat Setal (2017) Euglycemic diabetic ketoacidosis accompanied by severe hypophosphatemia during recovery in a patient with type 2 diabetes being treated with canagliflozin/metformin combination therapy. Clin Diabetes 35(4):249-251

15. Gaasbeek A, Meinders AE (2005) Hypophosphatemia: an update on its etiology and treatment. Am J Med 118(10):1094-1101

16. Choi HS et al (2018) Respiratory failure in a diabetic ketoacidosis patient with severe hypophosphatemia. Ann Pediatr Endocrinol Metab 23(2):103-106

17. Pfefferkorn F et al (2019) Diabetische Ketoazidose unter Empagliflozin. Swiss Med Forum. https://doi. org/10.4414/smf.2019.08247

18. Blau JE et al (2017) Ketoacidosis associated with SGLT2 inhibitor treatment: analysis of FAERS data. Diabetes Metab Res Rev 33(8):10.1002/dmrr.2924. https://doi.org/10.1002/dmrr.2924

19. Pozzilli P, Pieralice S (2018) Latent autoimmune diabetes in adults: current status and new horizons. Endocrinol Metab (Seoul) 33(2):147-159

20. Peters AL et al (2015) Euglycemic diabetic ketoacidosis:a potential complication of treatment with sodium-glucose cotransporter 2 inhibition. Diabetes Care 38(9):1687-1693

21. Erondu $\mathrm{N}$ et al (2015) Diabetic ketoacidosis and related events in the canagliflozin type 2 diabete clinical program. Diabetes Care 38(9):1680-1686

22. Fralick M, Schneeweiss S, Patorno E (2017) Risk of diabetic ketoacidosis after initiation of an SGLT2 inhibitor. N Engl J Med 376(23):2300-2302

23. Ueda P et al (2018) Sodium glucose cotransporte 2 inhibitors and risk of serious adverse events: nationwide register based cohort study. BMJ 363:k4365

24. Benoit SR et al (2018) Trends in diabetic ketoacidosis hospitalizations and in-hospital mortality-United States, 2000-2014. Mmwr Morb Mortal Wkly Rep 67(12):362-365

25. Haak T et al (2020) Therapie des Typ-1-Diabetes Kurzfassung der S3 Leitlinie (AWMF-registernummer: 057-013; 2. Auflage). Diabetol Stoffwechs 15(S01):S40-S50

26. Mahler SA et al (2011) Resuscitation with balanced electrolyte solution prevents hyperchloremic metabolic acidosis in patients with diabetic ketoacidosis. Am J Emerg Med 29(6):670-674

27. Jeon JY et al (2019) Clinical characteristics of diabetic ketoacidosis in users and non-users of SGLT2 inhibitors. Diabetes Metab 45(5):453-457
Strukturiertes Behandlungsprogramm bei rheumatoider Arthritis

Auch für rheumatoide Arthritis gibt es jetzt ein strukturiertes Behandlungsprogramm: Dieses soll durch koordinierte Betreuung und Schulung von Patienten dazu beitragen, Therapieziele zu erreichen.

Die Deutsche Gesellschaft für Rheumatologie e. V. (DGRh) begrüßt den Beschluss des Gemeinsamen Bundesausschuss, ein Disease-Management-Programm (DMP) für die rheumatoide Arthritis (RA) zu initiieren: Sie sieht darin eine große Chance für die Versorgung von Patienten mit RA. Denn wirksame Therapien können eine RA zum Stillstand bringen - vorausgesetzt, sie kommen rechtzeitig und gezielt zum Einsatz.

Um Patienten zu einer zügigen Diagnose und Behandlung zu verhelfen, definiert das Programm Schnittstellen zwischen Haus- und Fachärzten. Außerdem wird die Therapie beim niedergelassenen Arzt, im Krankenhaus oder einer Reha-Einrichtung koordiniert und stets an den individuellen Gesundheitszustand angepasst. Mithilfe von Patientenschulungen werden die Betroffenen zudem aktiv involviert und darin gestärkt, ihre chronische Erkrankung zu bewältigen und ihre Lebensqualität zu verbessern.

Nach der Definition von Inhalten und Prozessen müssen nun die praktischen Umsetzungen erfolgen und Verträge zwischen Krankenkassen, Praxen und Kliniken geschlossen werden. In etwa einem Jahr können sich erste Patienten in das Programm einschreiben.

Quelle: Deutsche Gesellschaft für Rheumatologie e. V. (DGRh), www.dgrh.de 
Hier steht eine Anzeige.

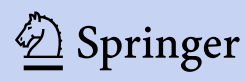

